\title{
Evidence for pearl-like magnetic island structures at dawn and dusk side magnetopause
}

\author{
W.-L. Teh and L.-N. Hau \\ Institute of Space Science, National Central University, Jhongli, Taiwan, R.O.C.
}

(Received February 16, 2004; Revised June 30, 2004; Accepted July 1, 2004)

\begin{abstract}
Two-dimensional magnetopause structure with a series of pearl-like magnetic islands at the dawn side of the equatorial plane was reported earlier by Hau and Sonnerup (1999) from the AMPTE/IRM data by solving the GradShafranov equation as a spatial initial value problem. This paper presents another rare event that appears like the image of the dawn event at the dusk side magnetopause based on the analysis of two years satellite data and similar reconstruction method. The two crossings occur at $(x, y, z)=(7.13,-6.64,0.4)$ and $(7.93,9.06,-1.89) R_{E}$ with magnetopause normal $\hat{\mathbf{n}}$ being $(0.85,-0.52,0.003)$ and $(0.82,0.55,-0.17)$ in the GSE coordinates, respectively. Both events are tangential discontinuity-like structures with shear angles being $124^{\circ}$ and $97^{\circ}$ and convect tailward with the deHoffmann-Teller frame velocity $\mathbf{V}_{\mathrm{HT}}$ of $(-141.88,-215.47,72.48)$ and $(-100.26,144.52,20.90)$ $(\mathrm{km} / \mathrm{s})$, respectively, that form approximately $90^{\circ}$ from $\hat{\mathbf{n}}$. The magnetic field configurations in both cases show great similarity with the same island width of $\sim 500 \mathrm{~km}$ and aspect ratio of $\sim 0.2$.
\end{abstract}

Key words: Magnetopause, current layer, reconnection, tearing-mode instability.

\section{Introduction}

Studies of magnetopause structure are the key to the understanding of solar terrestrial interaction processes. Observational evidences for magnetic field reconnection at the Earth's magnetopause have been overwhelming. The principal theoretical basis lies on the analysis of whether the plasma flow is approximately accelerated to the Alfvén speed in the deHoffmann-Teller (HT) frame, the so-called Walén relation associated with the rotational discontinuity (RD) current layer. As a recent example, Phan et al. (2001) reported an event detected by the Wind satellite at the low-latitude dawn tail magnetopause that shows the jetting plasma flow near the reconnection side being accelerated to 98\% of the Alfvén speed in the HT frame. Bi-directional jets near the reconnection site at the dawn side of the magnetopause flank have also been observed simultaneously by the Equator-S and Wind satellites (Phan et al., 2000). More recently, Mozer et al. (2002) established the evidence of diffusion region of about 6 magnetosheath ion skin depths $(\sim 600$ $\mathrm{km})$ at the subsolar magnetopause based on the Polar satellite data; the Hall magnetic as well as electric field signatures are also observed.

Aside from RD structure of open magnetosphere, the magnetopause may be of tangential discontinuity (TD)-like structure. Many theories and numerical simulations have shown that TD current layers may be unstable to the tearing-mode instability (TMI) that involves a series of magnetic islands or plasmoids separated by the $\mathrm{X}$ points (e.g., Hau and Chiou, 2001). The possible occurrence of TMI in the dayside magnetopause was addressed by Greenly and Sonnerup (1981).

Copy right(c) The Society of Geomagnetism and Earth, Planetary and Space Science (SGEPSS); The Seismological Society of Japan; The Volcanological Society of Japan; The Geodetic Society of Japan; The Japanese Society for Planetary Sciences; TERRAPUB.
The first direct evidence for the existence of a series of magnetic islands embedded within the magnetopause current layer was provided by Hau and Sonnerup (1999) from the AMPTE/IRM data based on the reconstruction method developed by Sonnerup and Guo (1996) and Hau and Sonnerup (1999). In particular, these authors were able to recover the two-dimensional equilibrium configuration from the plasma and magnetic field measurements taken by the single spacecraft traversing the magnetopause current layer. The robustness of the reconstruction technique has been shown by Hu and Sonnerup (2000) who utilized two spacecraft (AMPTE/IRM and UKS) data to reconstruct the magnetopause transect separately for the same event and showed that they are similar in the overall magnetic field topology. Recently, the reconstruction method was further improved by Hu and Sonnerup (2003) that may include two branches of current profiles for the plasmas on both sides of the magnetopause as well as the time-varying HT frame velocity, etc.

The Oct 19, 1984 event reported by Hau and Sonnerup (1999) has several unique properties in that it is an approximately one-dimensional TD with very small residual velocity in the HT frame and the HT velocity $\mathbf{V}_{\mathrm{HT}}$ is nearly along the magnetopause. The spacecraft is thus in a good situation of observing the imbedded series of magnetic islands convecting down the tail. This case is however rare in that in most crossings the magnetopause moves in and out along $\hat{\mathbf{n}}$ with large velocity for the satellite to resolve its detailed structure. In this paper, we adopt the reconstruction technique similar to that developed by Hau and Sonnerup (1999) to recover the magnetopause transect based on the analysis of two years AMPTE/IRM plasma and magnetic field data, with the purpose of searching for more evidence of pearllike magnetic island structure in the magnetopause. It is shown that the events of Oct 19, 1984 and Aug 8, 1985, 
located at the dawn and dusk sides of the dayside magnetopause flank, display very similar feature of a series of magnetic islands embedded within the TD magnetopause current layer. To assure the quality of analysis results, both methods of MVA (Sonnerup and Scheible, 1998) and minimization of Faraday residue (MFR) (Terasawa et al., 1996; Khrabrov and Sonnerup, 1998) are employed to obtain the magnetopause normal. The statistical bootstrap method (Kawano et al., 1995) along with the analytical error estimate for MVA (Khrabrov and Sonnerup, 1998a) are also implemented to estimate the errors associated with the magnetopause normal and the deHoffmann-Teller velocity. Both cases are shown to have high quality of MVA as well as HT frame that are suitable for the study of magnetopause reconstruction. The results based on two-branches fitting curves for the currents on both sides of the magnetopause are also discussed.

\section{Method Description}

We first briefly review the method used for recovering two-dimensional magnetic field and plasma maps of TD-like magnetopause structures. For detailed description of the reconstruction technique, it is referred to the paper by Hau and Sonnerup (1999). The problem is concerned about solving the Grad-Shafranov (GS) equation in two dimensions $(\partial / \partial z \approx 0)$ :

$$
\nabla^{2} A=-\mu_{0} \frac{d P_{t}}{d A}
$$

in the HT frame, for which $P_{t}=\left(P+B_{z}^{2} / 2 \mu_{0}\right)$ and $A(x, y)$ is the magnetic vector potential that is related to the magnetic field vector $\vec{B}$ by $\vec{B}=\nabla A \times \hat{z}+B_{z}(A) \hat{z}$. An important issue with the reconstruction scheme is then to find the correct invariant axis $\hat{z}$. To achieve this purpose, we need to first find the normal vector of the magnetopause and the HT frame velocity $\vec{V}_{\mathrm{HT}}$ and assume the invariant axis to be on the plane perpendicular to the magnetopause normal. The GS equation is then solved in the $(\hat{x}, \hat{y}, \hat{z})$ coordinates in which $\hat{x}=-\frac{\vec{V}_{H T}-\left(\vec{V}_{H T} \bullet \hat{z}\right) \hat{z}}{\left|\vec{V}_{H T}-\left(\vec{V}_{H T} \bullet \hat{z}\right) \hat{z}\right|}$ is in the direction of spacecraft trajectory projected on the plane perpendicular to the guessed invariant axis, $\hat{z}$, and $\hat{y}=\hat{z} \times \hat{x}$. The magnetic potential $A$ values along the $x$ axis are calculated by integration of $B_{y}=-\partial A / \partial x$ using $d x=-\vec{V}_{H T} \bullet \hat{x} d t$. Note that in this scheme the effect due to the curved trajectory of spacecraft caused by the small residual inward and outward motion of the magnetopause in the HT frame has been neglected. The plot of $P_{t}=\left(P+B_{z}^{2} / 2 \mu_{0}\right)$ versus $A$ is prepared to obtain the functional form of $P_{t}(A)$ and then the integration proceeds explicitly in $\pm \hat{y}$ direction starting at $y=0$ by implementing $B_{x}(x, 0)=\partial A /\left.\partial y\right|_{y=0}$ and $A(x, 0)$ as initial values. The criteria for choosing a suitable invariant axis are to optimize the consistency of $P=P(A)$ and $B_{z}=B_{z}(A)$ required for $2 \mathrm{D}$ equilibrium and to minimize scatter in the plot of $P_{t}(A)$ by mean of stepwise rotation of $\hat{z}$ around the normal vector.

\section{Dawn-Side Event: Oct 19, 1984}

Our study is based on the analysis of two years AMPTE/IRM plasma and magnetic field data with temporal resolution of $4.37 \mathrm{sec}$. The event of Oct 19, 1984, 0517:410519:48 UT, comprising 30 data points and occurring at $(7.13,-6.64,0.4) R_{E}$ in GSE coordinates, i.e., the dawn side of the dayside magnetopause in the equatorial plane, is an outbound crossing and has been studied in several papers (Sonnerup et al., 1990; Hau and Sonnerup, 1999). The HT analysis shows a high quality result with the correlation coefficient of 0.97 and the Walén analysis shows a small slope of -0.08 indicating a TD-like structure. Its normal vector based on the MVA is of $(0.85,-0.52,0.003)$ that points sunward and toward the dawnside and the normal magnetic field component $B_{n}$ is of $-0.33 \mathrm{nT}$. The normal vector based on the MFR analysis is of $(0.91,-0.39,0.119)$ that forms an angle of $10.7^{\circ}$ to the normal vector based on the MVA. We have calculated the normal uncertainty based on the following analytical error estimate method

$$
\left|\Delta \varphi_{i j}\right|=\sqrt{\lambda_{3}\left(\lambda_{i}+\lambda_{j}-\lambda_{3}\right) /\left((N-1)\left(\lambda_{i}-\lambda_{j}\right)^{2}\right)}
$$

$\left|\Delta<\vec{B} \bullet \hat{x}_{3}>\right|=\sqrt{\lambda_{3} /(N-1)+\left(\Delta \varphi_{32} \vec{B} \bullet \hat{x}_{2}\right)^{2}+\left(\Delta \varphi_{31} \vec{B} \bullet \hat{x}_{1}\right)^{2}}$ for which $\Delta \varphi_{i j}$ is the angular uncertainty of eigenvector $\hat{x}_{i}$ for rotation toward or away from eigenvector $\hat{x}_{j} ; N$ is the number of data points; $\lambda$ is the eigenvalue; $\hat{x}_{1}, \hat{x}_{2}$, and $\hat{x}_{3}$ are the maximum, intermediate, and minimum variance directions, respectively; and $\Delta<B_{n}>$ is the expected uncertainty of the normal magnetic component. For the Oct 19 , 1984 event, $\Delta \varphi_{31}, \Delta \varphi_{32}$ and $\Delta<B_{n}>$, are of $0.8^{\circ}, 3.09^{\circ}$ and $\pm 1.56 \mathrm{nT}$, respectively. When the bootstrap method is applied, the uncertainties associated with the magnetopause normal are of $\Delta \varphi_{31}=0.8^{\circ}, \Delta \varphi_{32}=3.15^{\circ}$, and $\Delta<B_{n}>= \pm 0.99 \mathrm{nT}$, in good agreement with the analytical error estimate. The deHoffmann-Teller velocity $\mathbf{V}_{\mathrm{HT}}$ of this event is $(-141.88,-215.47,72.48)(\mathrm{km} / \mathrm{s})(\mathrm{GSE})$, i.e., the plasma flow is tailward and toward the dawnside, and the magnetopause moves along $\hat{\mathbf{n}}$ with a velocity $\left(\mathbf{V}_{H T} \bullet \hat{\mathbf{n}}\right)$ of $-9.08 \mathrm{~km} / \mathrm{s}$. (Note that the negative sign is consistent with the outbound crossing.) These analysis results show that this TD event has high quality magnetopause normal as well as HT frame that we may proceed to perform the $2 \mathrm{D}$ reconstruction. In particular, the 2D structure is assumed to be in static MHD equilibrium in the HT frame and the invariant axis is assumed to be on the plane perpendicular to $\hat{\mathbf{n}}$. If the tried invariant axis is chosen to be the eigenvector corresponding to the intermediate eigenvalue based on MVA, the optimal invariant axis is found to rotate $-40^{\circ}$ from it; in particular, $\hat{\mathbf{z}}=(-0.508,-0.837,-0.203)$, is obtained such that $P_{t}(A)$, $p(A)$, and $B_{z}^{2}(A)$ have relatively high correlations with the magnetic vector potential $A$, where $P_{t}=p+B_{z}^{2} / 2 \mu_{0}$. Figure 1 shows the reconstructed field map on the plane perpendicular to the invariant axis, i.e., the contours of $A(x, y)$, that displays a series of magnetic islands embedded within the magnetopause structure. The colors denote the strength of the guide field, $B_{z}(A)$, that is strongest at the island center and in the three dimensional view flux rope structures can be seen clearly. Note that in the field map, the satellite trajectory is a straight-line located at $y=0$ and there exists a small residual flow (not shown here but can be found in the paper by Hau and Sonnerup) that has the component perpendicular to the local magnetic field, an indication that the frozen-influx condition does not comply everywhere. This is merely to point out that the existence of HT frame is not incompatible with the feature of magnetic field reconnection seen in the map. 


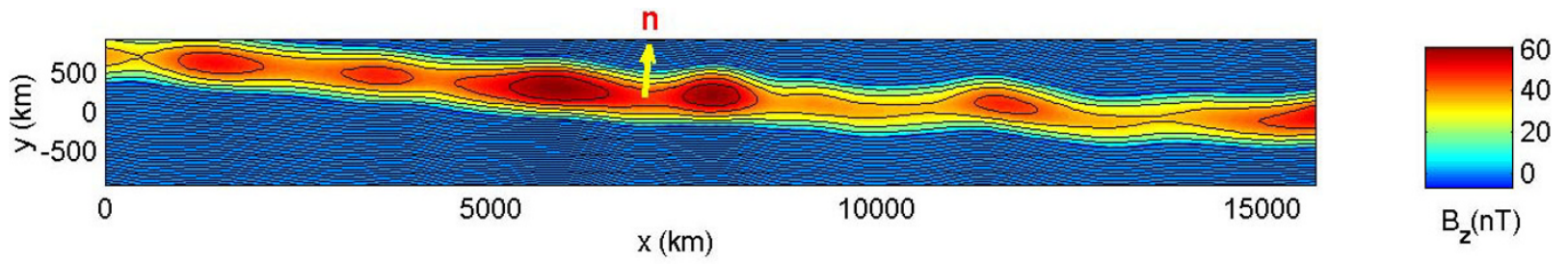

Dawn

Dusk

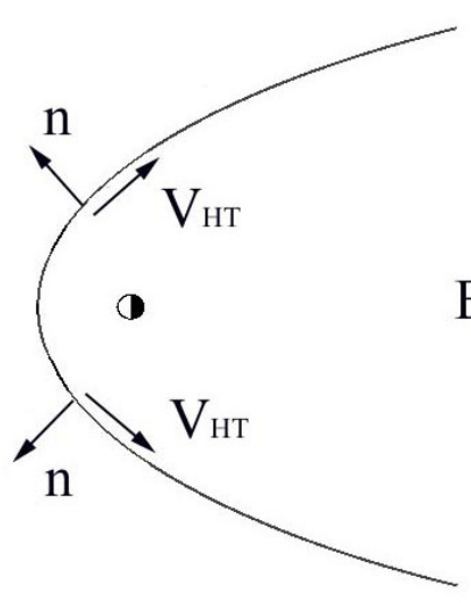

\section{Equatorial Plane}
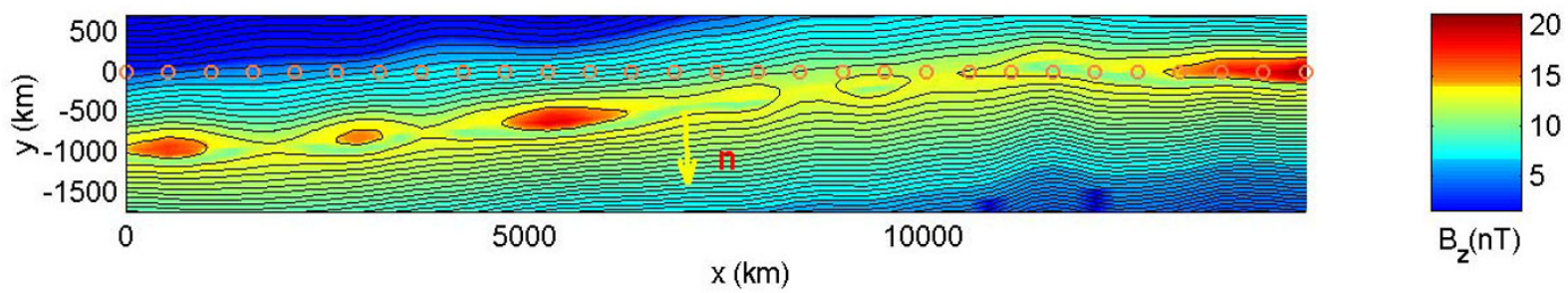

Fig. 1. The reconstructed field maps for Oct 19, 1984 and Aug 8, 1985 AMPTE/IRM events at the dawn and dusk sides of the dayside magnetopause in the equatorial plane. The solid lines are contours of magnetic vector potential $A(x, y)$ and the colors denote the strength of the guide field $B_{z}$. In the field maps the satellite trajectory is a straight-line located at $y=0$ with the data points denoted by circles and the left end of the maps corresponds to the starting point of the data interval in Fig. 2.
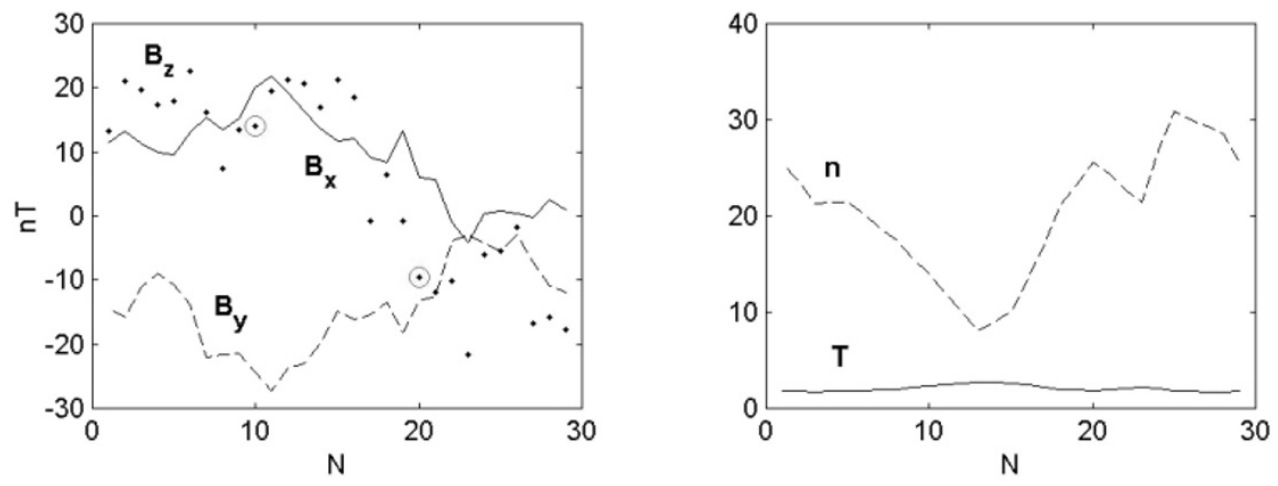

Fig. 2. Plots of three components of the magnetic field, $B_{x}, B_{y}, B_{z}(\mathrm{nT})$ in GSE coordinates, plasma number density (cm ${ }^{-3}$ ) and ion temperature $T$ $\left(\times 10^{6 \circ} \mathrm{K}\right)$ for the AMPTE/IRM Aug 8,1985 event. For comparison with the lower field map in Fig. 1 , data points $N=10$ and $N=20$ are marked by circles.

\section{Dusk-side Event: Aug 8, 1985}

Similar to the previous case, the event of Aug 8, 1985, during the interval 1338:23-1340:28 UT, is the last third of multiple magnetopause crossings and is also an outbound crossing that occurs at $(7.93,9.06,-1.89) R_{E}$, i.e., the dusk side of the dayside magnetopause, and comprises 29 data points. Figure 2 shows the plots of three magnetic field components, proton number density and temperature. The mag- netopause normal based on the MVA and MFR are, respectively, to be of $(0.82,0.55,-0.17)$ and $(0.80,0.57,-0.17)$, i.e., the difference is only $1.8^{\circ}$, even smaller than the previous case, and the $B_{n}$ based on MVA is of $-1.21 \mathrm{nT}$. The analysis of the normal uncertainties based on the analytical error estimate method gives rise to $\Delta \psi_{31}=0.63^{\circ}$, $\Delta \psi_{32}=1.83^{\circ}$ and $\Delta<B n>= \pm 0.45 \mathrm{nT}$, while the corresponding quantities based on the bootstrap method are of 

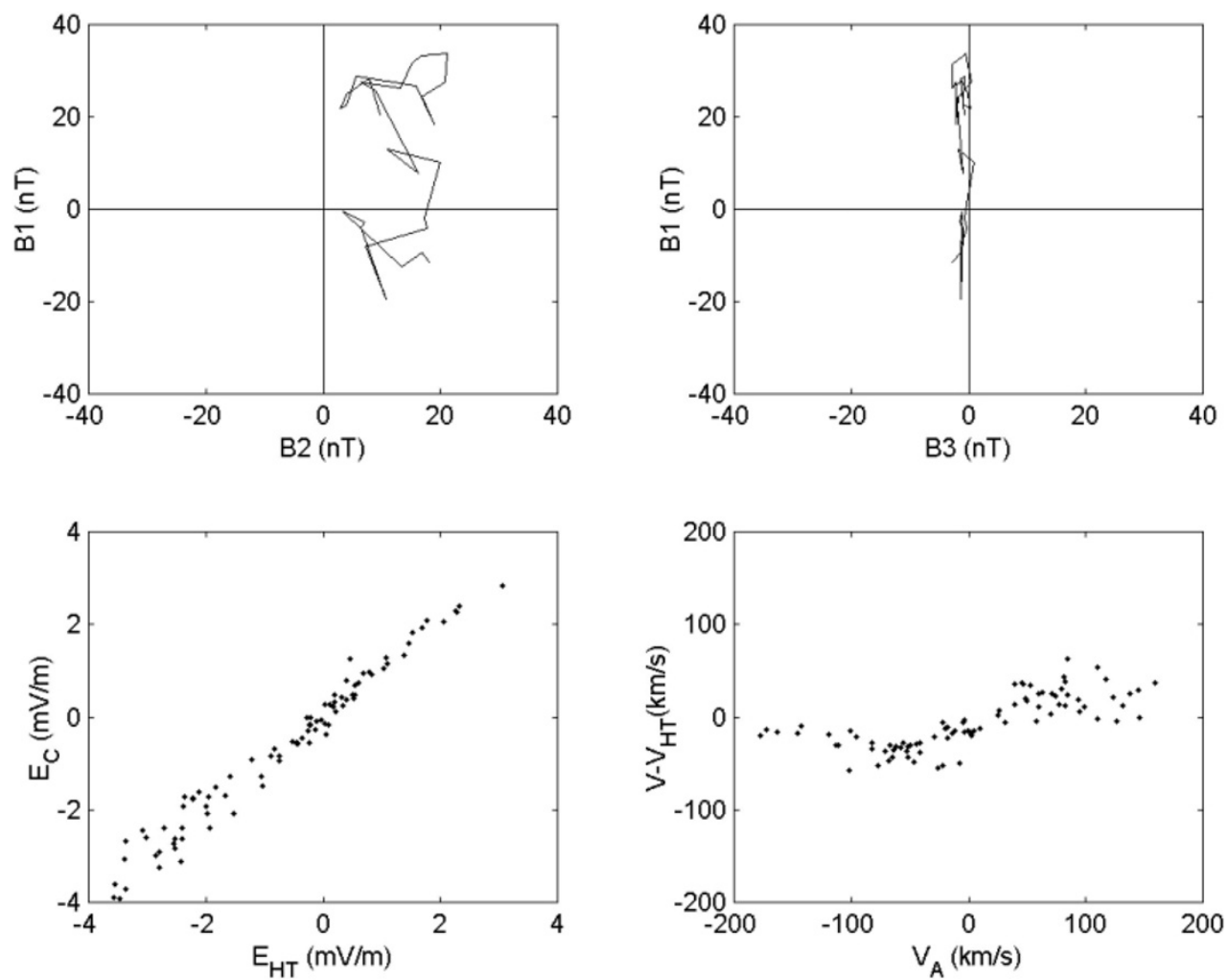

Fig. 3. Analysis results for the AMPTE/IRM Aug 8, 1985 event. The upper two panels are the magnetic hodogram pair from the MVA for which $B_{3}$ corresponds to the magnetopause normal component $B_{n}$; the left bottom panel shows the HT correlation with $\mathbf{V}_{\mathrm{HT}}=(-100.26$, 144.52, 20.90) $\mathrm{km} / \mathrm{s}$ and $R=0.98$; and the right bottom panel is the Walén correlation.
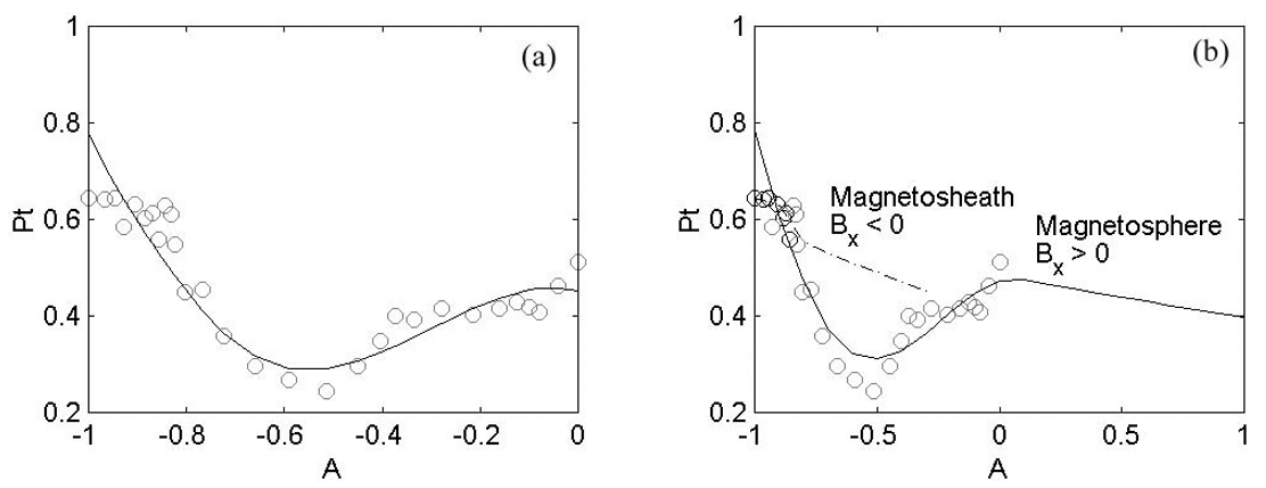

Fig. 4. The curves of $P_{t}=\left(p+B_{z}^{2} / 2 \mu_{0}\right)$ as a function of $A$ for the AMPTE/IRM, Aug 8,1985 event. The $P_{t}(A)$ curves are fitted with one branch and two branches curves in the left and right panels, respectively.

$\Delta \varphi_{31}=0.63^{\circ}, \Delta \varphi_{32}=1.78^{\circ}$ and $\Delta<B_{n}>= \pm 0.42 \mathrm{nT}$. The HT analysis shows high correlation coefficient of 0.98 , indicating the existence of a good HT frame, and the Walén analysis shows a small slope of 0.25 , indicating a TD-like magnetopause structure. While the magnetopause normal is pointing sunward and toward the duskside, the $\mathbf{V}_{\mathrm{HT}}$ is of $(-100.26,144.52,20.90)(\mathrm{km} / \mathrm{s})$, i.e., the plasma flow is tailward and toward the duskside and $\mathbf{V}_{\mathrm{HT}} \bullet \hat{\mathbf{n}}$ is of -5.82 $\mathrm{km} / \mathrm{s}$. This event is thus very similar to the Oct 19,1984 crossing in that the normal magnetopause motion is small and the satellite is in a unique situation of observing the details of the structure passing by. Figure 3 shows the analysis results for MVA, HT frame as well as Walén relation that are quite similar to those for the Oct 19, 1984 crossing (see figure 5 of Hau and Sonnerup, 1999).

In this event, the optimal invariant axis is $(0.2667,-0.6204,-0.7374)$ that is $20^{\circ}$ from the intermediate axis of MVA; the corresponding $P_{t}$ versus $A$ curve is shown in Fig. 4(a). Unlike the previous case the quality of $P_{t}(A)$ curve fitting as well as the field map of this event are not highly sensitive to the rotation angles of $\theta=20^{\circ}-40^{\circ}$. Nevertheless the $p(A)$ and $B_{z}^{2}(A)$ curves show better fitting for $\theta=20^{\circ}$. The reconstructed magnetopause transect maps shown in Fig. 1 indicate that both events occurring at the dawn and dusk sides of the magnetopause (whose shape is merely a sketch but not a quantitative model as 
Table 1. Summary of analysis results for both events.

\begin{tabular}{ccc}
\hline & October 19, 1984 (dawn) & August 8, 1985 (dusk) \\
\hline MP location & $(7.13,-6.64,0.4) R_{E}$ & $(7.93,9.06,-1.89) R_{E}$ \\
$\hat{\mathbf{n}}(\mathrm{MVA}), B_{n}$ & $(0.85,-0.52,0.003),-0.33 \mathrm{nT}$ & $(0.82,0.55,-0.17),-1.21 \mathrm{nT}$ \\
$\mathbf{V}_{\mathrm{HT}}$ & $(-142,-215,72) \mathrm{km} / \mathrm{s}$ & $(-100,145,21) \mathrm{km} / \mathrm{s}$ \\
$\hat{\mathbf{z}}$ & $(-0.51,-0.84,-0.203)$ & $(0.27,-0.62,-0.74)$ \\
$\alpha$ & $91.78^{\circ}$ & $92.03^{\circ}$ \\
Shear angle & $124^{\circ}$ & $97^{\circ}$ \\
\hline
\end{tabular}

Note that $\alpha$ is the angle between $\hat{\mathbf{n}}$ and $\mathbf{V}_{\mathrm{HT}}$.

that developed, for example, by Shue et al. (1997)) display similar feature in that a series of magnetic islands are imbedded within the current layers with the width of about $500 \mathrm{~km}$ and the axial magnetic field is strongest at the $\mathrm{O}$ points.

The above calculations assume $P_{t}(A)$ to have the same form on the magnetospheric and magnetosheath sides. Recently the method of 2D magnetopause reconstruction technique was improved by $\mathrm{Hu}$ and Sonnerup (2003) to allow two-branches $P_{t}$ curve fitting for both sides of the current layer. In particular, the scheme was applied to the Sep 21, 1984 and Oct 30, 1984 crossings that show distinct two branches of curves for $P_{t}$ versus $A$. To assure the quality of reconstruction results, we have also examined the possibility of two branches of $P_{t}(A)$ curves for both events. It is found that the Oct 19, 1984 case does not have a need to fit with two $P_{t}(A)$ curves, while for the Aug 8, 1985 case such a possibility may exist. As shown in Fig. 4(b), two branches of curves are fitted for $P_{t}$ as a function of $A$. It is seen that the data used for magnetosheath $\left(B_{x}<0\right)$ is quite less than the magnetosphere $\left(B_{x}>0\right)$. Note that the $P_{t}(A)$ curve is extrapolated in the way that its slope being the current declines away from the magnetopause current and the calculations show that the field maps are not sensitive to the detailed extrapolation. Using the same data interval as well as the same optimal invariant axis as for the one branch case (Fig. 4(a)), we have obtained the reconstructed magnetopause transect map for two-branches $P_{t}(A)$ as shown in Fig. 1 for the dusk event that shows only minor difference from the one branch result. In particular, in agreement with the finding by $\mathrm{Hu}$ and Sonnerup (2003), the two-branches fitting gives rise to slightly thin magnetic island width. The study provides a further check for the quality of the field maps shown in Fig. 1.

\section{Summary}

The AMPTE/IRM Oct 19, 1984 event is the first example used to demonstrate the occurrence of two-dimensional magnetic island structure embedded within the TD magnetopause current (Hau and Sonnerup, 1999). This paper shows that such remarkable features may exist in the dawn as well as dusk sides of the dayside magnetopause flank. The two reconstructed field maps presented in Fig. 1 appear like image events that display great similarities in the overall magnetic field topology as well as the island width of about 500 $\mathrm{km}$ and average aspect ratio (the ratio of island width to length) of $\sim 0.2$ etc. Both events have been carefully selected based on the criteria that the analysis results for the magne- topause normal as well as the deHoffmann-Teller frame must be of extremely high quality. The residual velocity in the HT frame must also be very small for the static equilibrium to be assumed in the reconstruction scheme. To assure the quality of the magnetopause normal, we have adopted both MVA as well as MFR methods and carried out the error estimates based on the analytical and statistical bootstrap methods. We have also applied the bootstrap method to the HT frame analysis and found consistent results.

Table 1 summarizes some properties of both events that indicate the dawn-dusk symmetry of the location, magnetopause normal $\hat{\mathbf{n}}$ and the HT velocity $\mathbf{V}_{\mathrm{HT}}$ etc. The angles, $\alpha$, between $\hat{\mathbf{n}}$ and $\mathbf{V}_{\mathrm{HT}}$ are approximately $90^{\circ}$ in both cases such that the spacecraft has the unique opportunity of observing the series of pearl-like magnetic islands imbedded within the TD structure and convecting tailward. In most cases the magnetopause merely moves in and out rapidly for the satellites to resolve the overall detailed structure. The $\mathbf{V}_{\mathrm{HT}}$ velocities in both events are consistent with the picture of solar wind-magnetosphere interaction in that the whole structures are convecting antisunward with the speed of about 200 $\mathrm{km} / \mathrm{s}$. The magnetic island structures imbedded within the tangential discontinuity may be interpreted as the nonlinear saturated state of tearing-mode instability as demonstrated recently by Hau and Chiou (2001) based on the resistive MHD simulations (the island width is approximately 10 ion gyroradii that the MHD theory may still be applicable). In particular, for magnetic Reynolds number of $R_{m}=10^{3}-10^{5}$ and isothermal energy equation the aspect ratio of magnetic islands is about 0.18 close to the observation (the calculations show that within the framework of resistive MHD tearing the aspect ratio depends mostly on the equation of state but is less sensitive to $R_{m}$ in the range of $10^{3}-10^{5}$ ). It is interesting to note from Table 1 that both events are of high shear angles with $124^{\circ}$ and $97^{\circ}$, respectively. Although the events reported in the present paper are quite rare possibly due to the observational view, the remarkable similarities shown in the dawn and dusk reconstructed field maps indicate that the magnetic island structure may not be an unusual feature of the magnetopause current layer.

Acknowledgments. This research is supported by the National Science Council of the Republic of China under grants NSC922111-M-008-012 and NSC93-2111-M-008-009 to National Central University. The authors would like to thank B. U. Ö. Sonnerup for helpful comments. 


\section{References}

Greenly, J. B. and B. U. Ö. Sonnerup, Tearing modes at the magnetopause, J. Geophys. Res., 86, 1305, 1981.

Hau, L.-N. and S.-W. Chiou, On the linear and nonlinear resistive tearing mode instability, J. Geophys. Res., 106, 8371, 2001.

Hau, L.-N. and B. U. Ö. Sonnerup, Two-dimensional coherent structures in the magnetopause: Recovery of static equilibria from single-spacecraft data, J. Geophys. Res., 104, 6899, 1999.

$\mathrm{Hu}, \mathrm{Q}$. and B. U. Ö. Sonnerup, Magnetopause transects from two spacecraft: A comparison, Geophys. Res. Lett., 27, 1443, 2000.

$\mathrm{Hu}$, Q. and B. U. Ö. Sonnerup, Reconstruction of two-dimensional structures in the magnetopause: Method improvements, J. Geophys. Res., 108, 1011, 2003.

Kawano, H. and T. Higuchi, The bootstrap method in space physics: Error estimation for the minimum variance analysis, Geophys. Res. Lett., 22 307, 1995.

Khrabrov, A. V. and B. U. Ö. Sonnerup, Orientation and motion of current layers: Minimization of the Faraday residue, Geophys. Res. Lett., 25, 2373, 1998 .

Khrabrov, A. V. and B. U. Ö. Sonnerup, Error estimates for minimum variance analysis, J. Geophys. Res., 103, 6641, 1998a.

Mozer, F. S., S. D. Bale, and T.-D. Phan, Evidence of diffusion regions at a subsolar magnetopause crossing, Phys. Rev. Lett., 89, 015002, 2002.

Phan, T.-D., L. M. Kistler, B. Klecker, G. Haerendel, G. Paschmann, B. U. Ö. Sonnerup, W. Baumjohann, M. B. Bavassano-Cattanneo, C. W. Carlson, A. M. Dilellis, K.-H. Fornacon, L. A. Frank, M. Fujimoto, E. Georgescu, S. Kokubun, E. Moeblus, T. Mukai, M. Øieroset, W. R.
Paterson, and H. Reme, Extended magnetic reconnection at the Earth's magnetopause from detection of bi-directional jets, Nature, 404, 848850, 2000.

Phan, T.-D., B. U. Ö. Sonnerup, and R. P. Lin, Fluid and kinetic signatures of reconnection at the dawn tail magnetopause: Wind observations, $J$. Geophys. Res., 106, 25,489, 2001.

Shue, J.-H., J. K. Chao, H. C. Fu, C. T. Russell, P. Song, K. K. Khurana, and H. J. Singer, A new functional form to study the solar wind control of the magnetopause size and shape, J. Geophys. Res., 102, 9497, 1997.

Sonnerup, B. U. Ö. and M. Guo, Magnetopause transects, Geophys. Res. Lett., 23, 3679, 1996.

Sonnerup, B. U. Ö. and M. Scheible, Minimum and maximum variance analysis, in Analysis Methods for Multi-Spacecraft Data, edited by G Paschmann and P. W. Daly, chap. 8, pp. 185-220, Int. Space Sci. Inst., Bern, Switzerland, and Eur. Space Agency, Paris, France, 1998.

Sonnerup, B. U. Ö., I. Papamastorakis, G. Paschmann, and H. Lühr., The magnetopause for large magnetic shear: Analysis of convection electric fields from AMPTE/IRM, J. Geophys. Res., 95, 10,541, 1990.

Terasawa, T., H. Kawano, I. Shinohara, T. Mukai, Y. Saito, M. Hoshino, A. Nishida, S. Machida, T. Nagai, T. Yamamoto, and S. Kokubun, On the determination of a moving MHD structure: Minimization of the residue of integrated Faraday's equation, J. Geomagn. Geoelectr., 48, 603, 1996.

W.-L. Teh (e-mail: waileong@jupiter.ss.ncu.edu.tw) and L.-N. Hau (email: lnhau@jupiter.ss.ncu.edu.tw) 